\title{
Increased Creatinine Clearance in Acute Kidney Injury due to Heat Stroke
}

\author{
Daichi Urabe $^{\text {a }}$, Yuki Kotani ${ }^{a}$, b, Toshihide Tsujimoto ${ }^{a}$
}

\begin{abstract}
Acute kidney injury (AKI) is associated with increased mortality and risk of chronic kidney disease (CKD). Whereas AKI is well known to occur frequently in patients with heat stroke, creatinine clearance levels of these patients have not been evaluated in literature. In this report, we present the case of a patient with increased creatinine clearance who developed AKI due to heat stroke. A 40-year-old male with no past medical history was admitted to the intensive care unit of our hospital due to AKI from heat stroke. Although he was diagnosed with stage $3 \mathrm{AKI}$ at admission, his creatinine clearance, measured by a 12-h urine collection, was highly elevated to $230 \mathrm{~mL} / \mathrm{min} / 1.73 \mathrm{~m}^{2}$. His serum creatinine was decreased to the baseline level $12 \mathrm{~h}$ after admission, with fluid resuscitation. This case can suggest that creatinine clearance might be underestimated, although serum creatinine levels are elevated in AKI due to heat stroke.
\end{abstract}

Keywords: Acute kidney injury; Heat stroke; Creatinine clearance

\section{Introduction}

Acute kidney Injury (AKI) is associated with increased mortality and risk of chronic kidney disease (CKD). Whereas AKI is well known to occur frequently in patients with heat stroke, no studies have examined potential changes in creatinine clearance in this patient population. We herein present the case of a patient with AKI due to heat stroke whose creatinine clearance was elevated.

\section{Case Report}

A 40-year-old male without past medical or family history pre-

Manuscript submitted January 23, 2018, accepted January 29, 2018

a Department of Critical Care Medicine, Japanese Red Cross Society Wakayama Medical Center, 4-20, Komatsubara-dori, Wakayama City, Wakayama Prefecture, 640-8558, Japan

${ }^{b}$ Corresponding Author: Yuki Kotani, Japanese Red Cross Society Wakayama Medical Center, 4-20, Komatsubara-dori, Wakayama City, Wakayama Prefecture, 640-8558, Japan.Email: dkivoar287@gmail.com

doi: https://doi.org/10.14740/jmc3007w sented to our hospital with nausea and generalized muscle pain after working outdoors on a hot summer day. On arrival, his rectal temperature was $37.6^{\circ} \mathrm{C}$, and his serum creatinine, aspartate aminotransferase and alanine aminotransferase levels were 2.58 $\mathrm{mg} / \mathrm{dL}, 68 \mathrm{IU} / \mathrm{L}$ and $82 \mathrm{IU} / \mathrm{L}$, respectively. His serum creatinine kinase (CK) was 237 IU/L. On physical examination, his tongue was dry; however, no other abnormal findings were observed. He was diagnosed with heat stroke and renal dysfunction and admitted to the intensive care unit. According to the Kidney Disease: Improving Global Outcomes (KDIGO) criteria, his renal dysfunction was classified as stage 3 AKI based on the Modification of Diet in Renal Disease equation. Fluid resuscitation successfully decreased serum creatinine level to $0.84 \mathrm{mg} / \mathrm{dL}$ by 12 $\mathrm{h}$ after admission. However, his creatinine clearance, measured by a 12 -h urine collection, was elevated to $230 \mathrm{~mL} / \mathrm{min} / 1.73 \mathrm{~m}^{2}$. He was discharged home from hospital on day three.

\section{Discussion}

The presented case can suggest that in patients with elevated serum creatinine due to heat stroke, creatinine clearance calculated by a 12 -h urine collection might also be elevated. The KDIGO criteria and severity classification for AKI have an important role in predicting mortality and CKD risk. Previous studies found that AKI was associated with increased short and long-term risk of death and increased risk of CKD and end-stage renal disease (ESRD) $[1,2]$. Conversely, one study reported that while AKI occurred in about $90 \%$ of heat stroke patients, more than $95 \%$ of the AKI patients were alive with serum creatinine levels that were recovered to baseline at the time of discharge [3]. Thus, renal function may not be impaired in heat stroke patients diagnosed with AKI.

Rhabdomyolysis is known to be associated with heat stroke, and cause AKI. CK level in this case was under 250 IU/L throughout the hospital stay. It was much lower than the reported cases of rhabdomyolysis [4, 5]. Thus, we consider that the AKI was not caused by rhabdomyolysis, but by hypovolemia from heat stroke. The elevation of serum creatinine is potentially caused by muscle injury by heat stroke, thereby meeting the AKI criteria [3, 6-9].

As shown in this patient, creatinine clearance is also known to be probably elevated in septic AKI, ultimately affecting dosing of drugs such as antibiotics. In septic AKI patients, renal function was reported not to decline as low as expected, which could lead to underestimation of glomerular filtration rate and insufficient drug dosing [10]. This phenomenon is termed aug- 
mented renal clearance in which renal function for solution clearance is elevated more than expected by visible laboratory data in critically ill patients $[11,12]$. Therefore, more sensitive biomarkers such as the measured creatinine clearance are needed.

\section{Conclusions}

The measured creatinine clearance might be elevated in AKI due to heat stroke, and renal function for solution clearance might be underestimated with estimated glomerular filtration rate.

\section{Conflict of Interest}

No potential conflict of interest relevant to this article was reported.

\section{References}

1. Luo X, Jiang L, Du B, Wen Y, Wang M, Xi X, Beijing Acute Kidney Injury Trial w. A comparison of different diagnostic criteria of acute kidney injury in critically ill patients. Crit Care. 2014;18(4):R144.

2. Rimes-Stigare C, Frumento P, Bottai M, Martensson J, Martling CR, Walther SM, Karlstrom G, et al. Evolution of chronic renal impairment and long-term mortality after de novo acute kidney injury in the critically ill; a Swedish multi-centre cohort study. Crit Care. 2015;19:221.

3. Satirapoj B, Kongthaworn S, Choovichian P, Supasyndh O. Electrolyte disturbances and risk factors of acute kidney injury patients receiving dialysis in exertional heat stroke. BMC Nephrol. 2016;17(1):55.
4. Melli G, Chaudhry V, Cornblath DR. Rhabdomyolysis: an evaluation of 475 hospitalized patients. Medicine (Baltimore). 2005;84(6):377-385.

5. El-Abdellati E, Eyselbergs M, Sirimsi H, Hoof VV, Wouters K, Verbrugghe W, Jorens PG. An observational study on rhabdomyolysis in the intensive care unit. Exploring its risk factors and main complication: acute kidney injury. Ann Intensive Care. 2013;3(1):8.

6. Raju SF, Robinson GH, Bower JD. The pathogenesis of acute renal failure in heat stroke. South Med J. 1973;66(3):330-333.

7. Woodrow G, Brownjohn AM, Turney JH. The clinical and biochemical features of acute renal failure due to rhabdomyolysis. Ren Fail. 1995;17(4):467-474.

8. Chen CY, Lin YR, Zhao LL, Yang WC, Chang YJ, Wu HP. Clinical factors in predicting acute renal failure caused by rhabdomyolysis in the ED. Am J Emerg Med. 2013;31(7):1062-1066.

9. Junglee NA, Di Felice U, Dolci A, Fortes MB, Jibani MM, Lemmey AB, Walsh NP, et al. Exercising in a hot environment with muscle damage: effects on acute kidney injury biomarkers and kidney function. Am J Physiol Renal Physiol. 2013;305(6):F813-820.

10. Bragadottir G, Redfors B, Ricksten SE. Assessing glomerular filtration rate (GFR) in critically ill patients with acute kidney injury - true GFR versus urinary creatinine clearance and estimating equations. Crit Care. 2013;17(3):R108.

11. Sime FB, Udy AA, Roberts JA. Augmented renal clearance in critically ill patients: etiology, definition and implications for beta-lactam dose optimization. Curr Opin Pharmacol. 2015;24:1-6.

12. Udy AA, Roberts JA, Shorr AF, Boots RJ, Lipman J. Augmented renal clearance in septic and traumatized patients with normal plasma creatinine concentrations: identifying at-risk patients. Crit Care. 2013;17(1):R35. 